\title{
SUDDEN CARDIAC ARREST DURING GENERAL ANESTHESIA IN A PATIENT WITH NO CARDIOVASCULAR DISEASE
}

\author{
Meltem Kipri, Sule Akin Enes, Esra Çalışkan, Asli Karsli, Anis Aribogan \\ Baskent University Medical Faculty, Department of Anesthesiology and Reanimation, \\ Ankara, TURKEY \\ Purpose of the study:
}

Sudden cardiac arrest secondary to general anesthesia is rare in individuals with no apparent cardiovascular disease. In this case report, a sudden cardiac arrest was presented which was developed in anesthesia induction due to spontaneous coronary artery dissection in a young and healthy patient undergoing septoplasty.

\section{Materyal and Methods:}

A 28 year- old woman with no history of systemic illnesses or cardiac diseases underwent septoplasty Following anesthesia induction and endotracheal intubation, a difficult ventilation developed due to severe bronchospasm and pulmonary edema. Sudden cardiac arrest with ventricular fibrillation occured.CPR was started immediately and the patient was defibrillated according to recommendations of the ERC guidelines. Return of spontaneous circulation was performed in 35 minutes

Dissection and recannulation of the left main coronary artery was observed in percutaneous coronary invertention but placement of coronary stent was not indicated. Echocardiographia showed low ejection fraction $(E F<20 \%)$

Amiodarone and inotropic drugs were received the patient with mechanical ventilation and Targeted Temperature Management administration.

The patient was extubated in 36 hours with effective respiration and normal hemodynamic parameters (EF>30\%) and no neurologic deficit.No arrhythmia was seen in 12- leads ECG.

The patient completely recovered in 5 days. After 30 days,coronary angiography was re-performed and no coronary abnormality was seen.

\begin{abstract}
Result:
Abnormality of coronary arteries is common cause of sudden cardiac arrest in young adults In our patient sudden cardiac arrest developed by structural deformation ogf the left main coronary artery during the anesthesia induction.
\end{abstract}

\section{Conclusion}

Pathologies of coronary arteries should be evaluated in patients with sudden cardiac arrest under anesthesia regardless of age and current health status.

We considered that all the anesthesiologist in our department had taken ERC courses which was very important in successful resuscitation of the patient.

\section{Reference}

Rev Bras Anestesiol. 2012 62:87.

ERC Guidelines for Resuscitation 2015:p99. 